\title{
4.4 Gesundheitsverhalten und Lebensstile
}

Ebenso wie in anderen Bereichen können sich Menschen auch in Bezug auf ihre Gesundheit unterschiedlich verhalten. Solches Gesundheitsverhalten kann sich positiv oder negativ auf die Gesundheit auswirken. Selbst Verhaltensweisen, die nicht direkt auf die Gesundheit eines Menschen ausgerichtet sind, können die Gesundheit beeinflussen: So kann sich Stress z. B. entscheidend auf die gesundheitsbezogene Lebensqualität eines Menschen auswirken. Der Erwerb von Gesundheitskompetenz, d.h. von individuellen Fähigkeiten, die es ermöglichen, förderlich mit der eigenen Gesundheit und der Gesundheit Anderer umzugehen, kann zu einem persönlichen Gesundheitsgewinn und einer Verbesserung der Rahmenbedingungen für Gesundheit führen. In diesem Abschnitt definieren wir zuerst den Begriff des Gesundheitsverhaltens und betrachten drei Public Health-relevante Erklärungsmodelle für Gesundheitsverhalten. Wir beschäftigen uns mit einem häufig verwendeten Stressmodell, gehen den möglichen Ursachen von Stress nach, erfahren etwas über die durch Stress entstehenden volkswirtschaftlichen Kosten und über Methoden zum adäquaten Umgang mit Stress. Abschließend gehen wir näher auf das Konzept des gesundheitsrelevanten Lebensstils ein und beschäftigen uns mit den verschiedenen Formen von Gesundheitskompetenz.

Schweizerische Lernziele: CPH 1, CPH 33-36, CPH 65

\subsubsection{Modelle des Gesundheitsverhaltens}

\section{Reinhard Fuchs}

\section{Was ist Gesundheitsverhalten?}

Man unterscheiden zwei Arten von Gesundheitsverhalten:

- Positives Gesundheitsverhalten schützt und stärkt die Gesundheit eines Menschen, es bewahrt seine Unversehrtheit. Verhaltensweisen, die sich positiv auf die Gesundheit auswirken, reichen von regelmäßiger körperlicher Bewegung, ausgewogener Ernährung und der Teilnahme an Vorsorgeuntersuchungen bis hin zur Verwendung von Sonnenschutzmitteln oder aktivem Erholungsverhalten (Urlaubsgestaltung, Yoga, Meditation etc.).

- Durch gesundheitliches Risikoverhalten setzt sich eine Person einer erhöhten gesundheitlichen Gefährdung aus. Beispiele für ein solches Verhalten sind z. B. das Rauchen, der übermäßige Alkoholkonsum oder der Gebrauch „harter" Drogen.

Unter Gesundheitsverhalten im positiven Sinne versteht man also all jene Aktivitäten einer Person, die der Prävention von Krankheiten, der Förderung der Gesundheit und dem Schutz vor Verletzungen dienen. Gesundheitsverhalten ist Teil des gesundheitsrelevanten Lebensstils (s. Kap. 4.4.3).

Es sind v.a. fünf Gesundheitsverhaltensweisen („Big Five“), die für das Krankheits- und Sterbegeschehen in der Bevölkerung von entscheidender Bedeutung sind:

- Körperliche Bewegung

- Ernährungsgewohnheiten 
- Tabakkonsum

- Alkoholkonsum

- Schlafverhalten

Wir wissen heute, dass die Entstehung und der Verlauf der meisten chronischen Krankheiten (insbesondere von Herz-Kreislauf-Erkrankungen, bösartigen Tumoren, Diabetes mellitus und Rückenschmerzen) wesentlich durch das Gesundheitsverhalten der Menschen beeinflusst wird. Es stellt sich daher die Frage, welche Möglichkeiten es gibt, gesundheitsförderndes Verhalten zu stabilisieren und gesundheitliches Risikoverhalten im Sinne von Gesundheitsförderung zu reduzieren. Um diese Frage zu beantworten und später wirkungsvolle Interventionen einleiten zu können, ist es nötig, ein Verständnis dafür zu entwickeln, welche personalen, sozialen und strukturellen Faktoren das Gesundheitsverhalten steuern.

\section{Erklärungsmodelle des Gesundheitsverhaltens}

In den letzten Jahrzehnten wurden unterschiedliche Modelle zur Erklärung des Gesundheitsverhaltens entwickelt. Im Folgenden werden drei Public Health-relevante psychologische Erklärungsmodelle des Gesundheitsverhaltens näher vorgestellt. In diesem Zusammenhang sei auch auf das Modell gesundheitsrelevanter Lebensstile verwiesen (s. Kap. 4.4.3), das gesundheitsrelevantes Verhalten mit den vorhandenen gesundheitsbezogenen Orientierungen und sozialen Ressourcen in Verbindung setzt.

Transtheoretisches Modell: Das Transtheoretische Modell (TTM) wurde ursprünglich im Bereich der Raucherentwöhnung entwickelt, wird aber heute auch auf eine Vielzahl anderer Gesundheitsverhaltensweisen angewendet. Dieses Modell unterscheidet beim Prozess der Verhaltensänderung fünf Stadien bzw. Motivationsstufen (s. Box 4.4.1).

Entscheidend für das Verständnis dieses Modells ist es, dass es auf jeder dieser Motivationsstufen zu einem Rückschritt auf eine der vorangehenden Stufen kommen kann. Für unser Beispiel hieße dies: Acht Tage nachdem Frau B mit dem Rauchen aufgehört hat, erleidet sie einen Rückfall und raucht wieder eine Zigarette. Sie ist damit auf eine der vorangehenden Stufen zurückgefallen. Die fünf Stadien der Verhaltensänderung werden in Form einer Spirale dargestellt, auf der es für die betroffene Person Bewegungsmöglichkeiten sowohl in Richtung eines Fortschritts hin zu einer geplanten Verhaltensänderung als auch in Richtung eines Rückschritts gibt.

Verschiedene Strategien (zehn Prozesse, s. Internet-Ressourcen auf unserer LehrbuchHomepage) können betroffenen Personen nun dabei helfen, von einem Stadium ins nächste zu kommen. Das TTM besitzt damit eine hohe praktische Relevanz. Es ermöglicht, stadienspezifisch Zielgruppen zu identifizieren und für diese dann spezifisch abgestimmte Interventionen zu entwickeln. Frau B aus unserem Beispiel sollte also mit Hilfe einer für ihre Situation passenden Strategie darin unterstützt werden, dauerhaft mit dem Rauchen aufzuhören.

Theorie der Schutzmotivation: Die Theorie der Schutzmotivation (TSM) wurde entwickelt, um die Wirkung von abschreckenden Botschaften, so genannten Furchtappellen (Fear Appeals), auf das Gesundheitsverhaltens zu untersuchen. Dieses Modell geht 
Box 4.4.1: Das Transtheoretische Modell.

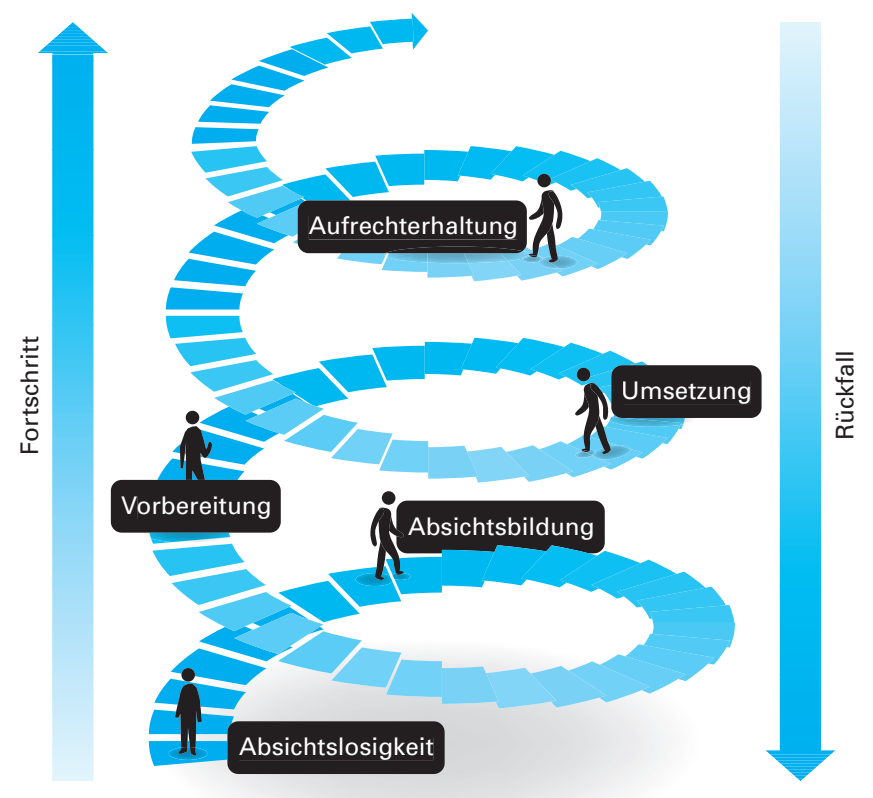

Das Transtheoretische Modell unterscheidet beim Prozess der Verhaltensänderung fünf Stadien, die hier am Beispiel der Raucherentwöhnung bei Frau B erläutert werden:

- Absichtslosigkeit: Frau B denkt nicht darüber nach, mit dem Rauchen aufzuhören.

- Absichtsbildung: Frau B überlegt, mit den Rauchen aufzuhören, hat aber noch keinen festen Vorsatz gefasst.

- Vorbereitung: Frau B hat sich fest vorgenommen, mit dem Rauchen aufzuhören. Sie versucht, ein paar Tage ohne Zigaretten auszukommen.

- Umsetzung oder Handlung: Frau B verzichtet ganz auf das Rauchen.

- Aufrechterhaltung: Das Nicht-Rauchen wird für Frau B zur Gewohnheit.

davon aus, dass gesundheitsrelevante Informationen, wie z. B. Warnhinweise auf Zigarettenpackungen, zwei Bewertungsprozesse in Gang setzen. Der Empfänger dieser Botschaft versucht zum einen den Grad der Bedrohung einzuschätzen, zum anderen beurteilt er die Möglichkeit der Bewältigung dieser Bedrohung. Abhängig vom Ergebnis dieser Einschätzungen wird er eine unterschiedlich starke Motivation zu protektivem Verhalten entwickeln („Schutzmotivation“). Bei der Bedrohungseinschätzung findet eine Kosten-Nutzen-Abwägung darüber statt, ob ein bestimmtes Gesundheitsverhalten (z. B. das Rauchen) begonnen, aufrechterhalten oder aufgegeben werden soll. Auf Sei- 
ten der Kosten wird der Schweregrad der Bedrohung bewertet, auf Seiten der Nutzen die erwarteten Vorteile des Verhaltens. Auch bei der Bewältigungseinschätzung findet eine Abwägung statt. Hier stehen auf der einen Seite Handlungswirksamkeit und Selbstwirksamkeit, auf der anderen Seite die Handlungskosten (s. Tab. 4.2). Mit dieser Theorie der Schutzmotivation wurde erstmals eine empirisch überprüfbare Modellvorstellung vom Wechselspiel der Risiko- und Ressourcenwahrnehmung bei der Entstehung von Gesundheitsmotivation entwickelt.

Talb. 4.2: Erläuterung der Theorie der Schutzmotivation am Beispiel von Warnhinweisen auf Zigarettenpackungen.

\begin{tabular}{lll}
\hline & Nutzen & Kosten \\
\hline Bedrohungseinschätzung: & $\begin{array}{l}\text { "Rauchen hilft mir, mit dem } \\
\text { Stress besser fertig zu } \\
\text { werden." }\end{array}$ & $\begin{array}{l}\text { "Wie gefährdet bin ich, an } \\
\text { Lungenkrebs zu erkranken?" }\end{array}$ \\
$\begin{array}{l}\text { Bewältigungseinschätzung: } \\
\text { - Handlungswirksamkeit }\end{array}$ & $\begin{array}{l}\text { "Wenn ich mit dem Rauchen } \\
\text { aufhören, kann ich dann } \\
\text { mein Krebsrisiko verringern?" }\end{array}$ & $\begin{array}{l}\text { "Wie anstrengend wäre es } \\
\text { für mich, mit dem Rauchen } \\
\text { aufzuhören?" }\end{array}$ \\
& "Würde ich es überhaupt & schaffen, mit dem Rauchen \\
aufzuhören?" & \\
\hline
\end{tabular}

Prozessmodell gesundheitlichen Handelns: Das Prozessmodell gesundheitlichen Handelns (Health Action Process Approach, HAPA) unterteilt den Vorgang, der zu einer Änderung des Gesundheitsverhaltens führen soll, in eine so genannte präintentionale Motivationsphase, die in der Formulierung spezifischer Verhaltensabsichten ihren Abschluss findet, und eine Phase, in der diese Verhaltensabsicht in tatsächliches Verhalten umgesetzt wird (postintentionale Volitionsphase ${ }^{17}$ ).

Die erste Phase der Verhaltensänderung wird von drei Faktoren geprägt:

- der Wahrnehmung von Gefährdungen bzw. Risiken (Risikowahrnehmung),

- der Ergebniserwartungen und

- der Überzeugung, das beabsichtigte Verhalten auch erfolgreich ausüben zu können (Selbstwirksamkeit).

Selbstwirksamkeit ist auch in der Umsetzungsphase von Bedeutung. Hinzu kommen aber auch noch die Faktoren der Handlungs- und Bewältigungsplanung. Im Verlauf der Handlungsplanung (Action Planning) werden einfache Was-Wann-Wo-Pläne erstellt. Bei der Bewältigungsplanung (Coping Planning) werden schließlich Strategien entwickelt, mit deren Hilfe innere oder äußere Verhaltensbarrieren umgangen werden können. Es ist das Verdienst des HAPA-Modells, das Augenmerk der Forschung auf den Umsetzungsprozess bei der Änderung von Gesundheitsverhalten gelenkt zu haben.

$\overline{17 \text { Volition: Prozess }}$ der Realisierung von Absichten 


\section{Gesundheitsverhalten am Beispiel Bewegung}

Die vorgestellten Gesundheitsverhaltensmodelle sind im Rahmen empirischer Studien mit Erfolg zur Erklärung von Bewegungs- und Ernährungsverhalten herangezogen worden. So sind z. B. Patienten, die während einer stationären Rehabilitation dazu aufgefordert werden, sich bezüglich ihres Bewegungsverhaltens detaillierte Handlungspläne („Welchen Sport werde ich zu Hause wann, wo und mit wem ausführen?") und Bewältigungspläne („Wie werde ich mit den zu erwartenden Handlungsbarrieren umgehen?") zurechtzulegen, nach der Klinikentlassung signifikant körperlich aktiver als die Patienten ohne eine solche Planungsintervention (s. Fallbeispiel in Web-Box 4.4.1 auf unserer Lehrbuch-Homepage).

In der Praxis erwiesen sich v.a. die Selbstwirksamkeitsüberzeugungen und Konsequenzerwartungen als kritische Punkte, z. B. bei der Motivation zur Sportteilnahme oder zum Wechsel auf eine gesündere Ernährungsweise. Es zeigte sich auch, dass für die Umsetzung dieser Motivation in konkretes Handeln bestimmte Fähigkeiten zur Selbststeuerung (Volition) eine zentrale Rolle spielen. Besonders wichtig sind in diesem Zusammenhang die Fähigkeit zur Entwicklung von realistischen Handlungsplänen (WasWann-Wo-Pläne) und Strategien zur Abschirmung der Pläne gegenüber möglichen Störeinflüssen (Barrierenmanagement). Vergleichende Interventionsstudien konnten inzwischen zeigen, dass das Gesundheitsverhalten durch die Beeinflussung solcher motivationaler und volitionaler Parameter entscheidend verändert werden kann.

Mittlerweile gibt es zahlreiche Bewegungsprogramme, z. B. auch zum Stressabbau (s. Kap. 4.4.2), die bei ihrer Umsetzung Erkenntnisse aus den vorgestellten Gesundheitsverhaltensmodellen berücksichtigen.

\subsubsection{Stress und Stressbewältigung}

\section{Heinz Bolliger-Salzmann}

Bei der Betrachtung des Gesundheitsverhaltens spielt das Thema Stress heute eine ganz besondere Rolle. Seine Bedeutung im Bereich der Gesundheitsförderung zeigt sich sowohl auf der individuellen als auch auf der gesellschaftlichen Ebene. Insbesondere in den industrialisierten Ländern hat die Komplexität der Lebenssituationen in den letzten Jahrzehnten erheblich zugenommen. Viele Menschen fühlen sich dadurch zunehmend belastet und immer häufiger auch überfordert. Ein solcher „negativer Stress" (Dis-Stress) kann langfristig krank machen.

\section{Definition und Ursachen von Stress}

Stress tritt dann auf, wenn es zu einem Missverhältnis zwischen den Anforderungen, die an eine Person gestellt werden, und den Möglichkeiten und Fähigkeiten dieser Person, die Anforderungen zu kontrollieren bzw. zu bewältigen (Coping) kommt. Als Stressoren oder Stressfaktoren bezeichnet man innere und äußere Reize, die auf den Menschen einwirken und eine Anpassungsreaktion von ihm erfordern. In einer Arztpraxis ist Stress z. B. sowohl auf Seiten der Patientlnnen als auch auf Seiten der Ärztlnnen zu finden. So kann die Ungewissheit über den eigenen Krankheitsverlauf bei einem Patienten situativ bedingten Stress auslösen, während bei vielen Ärztlnnen Stress z. B. durch das Überbringen einer sehr ungünstigen Prognose hervorgerufen wird. Äußere 
Stressoren können physikalischer (z. B. Lärm) oder sozialer Art sein (z. B. Probleme in der Beziehung), auch Überforderung und Zeitdruck gehören dazu. Persönlichkeitsbedingte Stresssituationen entstehen häufig bei Menschen, die über ungenügende Problemlösungskompetenzen verfügen, die perfektionistisch sind oder denen ein starkes Kontrollbedürfnis eigen ist. Ob bei einem Menschen jedoch letztendlich Stress entsteht, hängt entscheidend von der individuellen Bewertung durch den Betroffenen selbst ab. Eine Situation erzeugt insbesondere dann Stress, wenn sich ein Mensch dem hilflos ausgeliefert fühlt, wenn er keine Möglichkeit für sich sieht, etwas daran zu ändern.

Stress ist nicht an sich schlecht, im Gegenteil: Eine gemeisterte Herausforderung wie z.B. eine bestandene Prüfung oder eine sportliche Leistung sind Erfolgserlebnisse, die Menschen motivieren und beflügeln. Solche Gegebenheiten (Daily Uplifts) lösen so genannten positiven Stress (Eu-Stress) aus, der für das psychische Wohlbefinden äußerst wünschenswert ist. Die ihn hervorrufenden Stressoren wirken im Allgemeinen nur kurz. Stress wird erst dann zum gesundheitlichen Problem, wenn es sich um chronifizierten Dis-Stress handelt. In einem hektischen Alltag kann es hierzu jedoch recht schnell kommen, da sich die Wirkungen der zahlreichen Mikrostressoren (Daily Hassels, s. dazu auch den Web-Cartoon 4.4.1 „Stresstest“ auf unserer Lehrbuch-Homepage) aufsummieren.

\section{Das transaktionale Stressmodell}

Dieses Modell beschreibt Stresssituationen als komplexe Wechselwirkungsprozesse zwischen den Anforderungen einer Situation und der darin handelnden Person. Die betroffenen Personen nehmen dabei zwei kognitive Einschätzungen eines Stresszustandes vor. In einer primären Einschätzung bewerten sie die Situation („Umweltvariablen“). In der sekundären Einschätzung werden dann die persönlichen Bewältigungsmöglichkeiten bewertet („Personenvariablen“; s. Abb. 4.4).

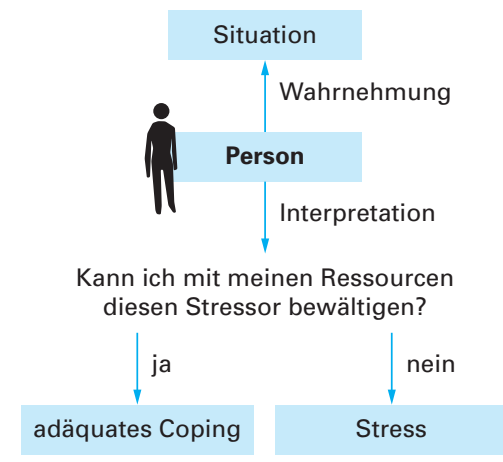

Abb. 4.4: Stark vereinfachtes Modell der transaktionalen Stressreaktion.

Da sich bei diesem Prozess eine denkende, fühlende und handelnde Person in einer sich verändernden Situation befindet, wird der Vorgang als „Transaktion“ bezeichnet. Die Person-Umwelt-Beziehung wird von der betroffenen Person dabei als herausfordernd, bedrohlich oder schädigend erlebt. Zur Überwindung von Problemsituationen stehen ihr jetzt grundsätzlich zwei Möglichkeiten offen: 
- Verfügt die Person über keine oder nur ungenügende Ressourcen (s. Kap. 4.1), gerät sie in Stress.

- Stehen ihr dagegen Möglichkeiten der Stressbewältigung zur Verfügung (adäquates Coping), kann dies auf verschiedene Weise geschehen. Je nach Art der vorhandenen Ressourcen bezeichnet man das dann angewandte Coping als problemorientiert, emotionsregulierend oder bewertungsorientiert.

\section{Kosten von Stress}

Welche Bedeutung Stress in volkswirtschaftlicher Hinsicht haben kann, zeigt eine 2003 veröffentlichte Studie des Schweizerischen Staatssekretariats für Wirtschaft (SECO), in der die stressbedingten Kosten am Arbeitsplatz für die Schweiz mit ca. 4,2 Mrd. CHF (medizinische Kosten: ca. 1,4 Mrd. CHF, Selbstmedikation gegen Stress: 348 Mio. CHF, Kosten im Zusammenhang mit Fehlzeiten und Produktionsausfall: 2,4 Mrd. CHF) angegeben wurden. Dies entsprach zum damaligen Zeitpunkt 1,2\% des schweizerischen Bruttoinlandproduktes. In der EU geht man davon aus, dass dort stressbedingte Kosten am Arbeitsplatz in Höhe von 5 bis $10 \%$ des Bruttosozialproduktes entstehen (vgl. auch Kap. 6.3.1).

Nach Angaben der Deutschen Rentenversicherung (2006) stehen in Deutschland psychische Erkrankungen - meist als Folge von Stress - mit über $30 \%$ an der Spitze der Ursachen für eine Frühberentung. Gesundheitsökonomisch relevant ist zudem, dass heute nahezu die Hälfte aller Krankheiten und somit auch ein erheblicher Teil der Krankheitskosten mit Stress in Zusammenhang gebracht werden können.

\section{Umgang mit Stress}

Die Web-Tab. 4.4.1 auf unserer Lehrbuch-Homepage zeigt Beispiele für Situationen, in denen Menschen unterschiedlichen Lebensalters mit positivem Stress (Eu-Stress) und negativem Stress (Dis-Stress) konfrontiert sind. Sie machen deutlich, dass Anspannung und Stress zu unserem Leben gehören.

Natürlich ist es sinnvoll, pathogenem Stress vorzubeugen. Wenn Stress jedoch nicht vermeidbar ist, dann ist ein regelmäßiger Ausgleich wichtig, um Gesundheitsgefahren erst gar nicht entstehen zu lassen. Insbesondere für Berufstätige in Gesundheitsberufen, die oft auch als Vorbild gesehen werden, ist ein adäquater Umgang mit Stress von großer Bedeutung. Dieser adäquate Umgang ist lernbar. Die dazu nötige Grundgelassenheit im Alltag kann man sich mit einer entsprechenden Technik aneignen.

Die meisten Menschen wenden stressreduzierende Maßnahmen in akuten Stresssituationen unbewusst an oder führen Alltagsaktivitäten aus, die beim Stressabbau behilflich sein können, ohne diese konkret zu planen (s. Web-Box 4.4.2 auf unserer Lehrbuch-Homepage). Regelmäßige Entspannung kann so zu einer Steigerung des Wohlbefindens und damit auch der Lebensqualität führen. Die beiden letzten Beispiele der Aufzählung (eine Zigarette rauchen/ein Glas Wein trinken) zeigen jedoch, dass es Entspannungsmethoden gibt, die sich bei fortgesetzter Anwendung negativ auch auf die Gesundheit auswirken können. 
Beispiele für sog. systematische Methoden zur Stressreduktion sind:

- Psychotherapeutische und/oder medizinische Begleitung

- Professionelles Coaching durch eine Fachperson

- Mentale Methoden (z.B. Alpha-Relaxing, BodyScan)

- Fernöstliche Methoden (z. B. Yoga, Tai Chi, Qi Gong)

- Körperbetonte Methoden (z.B. Progressive Muskelrelaxation)

- Suggestive Methoden (Autogenes Training)

- Psychoedukative Methoden (Biofeedback)

- Programme, wie z.B. das Stressimpfungstraining (SIT) nach Meichenbaum

Die Evidenzen für die Wirksamkeit der hier genannten Methoden sind unterschiedlich. Empirische Befunde legen aber nahe, dass sie dann wirksam sein können, wenn sie regelmäßig über einen gewissen Zeitraum und unter professioneller - psychotherapeutischer oder auch ärztlicher - Begleitung durchgeführt werden.

\subsubsection{Gesundheitsrelevante Lebensstile}

\section{Thomas Abel}

Stress, Zigarettenrauchen, Alkohol- und Medikamentenkonsum, die Art der Ernährung, das Maß an körperlicher Aktivität, das Sexualverhalten und vieles andere mehr beeinflussen in hohem Maße die Wahrscheinlichkeit eines Menschen, gesund zu sein. Für die meisten Industriestaaten ist inzwischen die wachsende Bedeutung solcher Verhaltenseinflüsse auf die individuelle und kollektive Gesundheit nachgewiesen. Gesundheitsrelevante Verhaltensweisen sind unmittelbar mit dem sozialen Kontext der Menschen verbunden. Je nach der sozialen Lage und dem Milieu, in denen sich ein Mensch befindet, stehen ihm dazu jedoch unterschiedliche Wahlmöglichkeiten zur Verfügung. Zwar sind die Muster der Lebensführung vom Einzelnen selbst gewählt worden, was aber tatsächlich gewählt werden kann, ist immer von den vorhandenen Ressourcen (s. Kap. 4.1) abhängig und wird von den Normen und Werten, die in den jeweiligen Bezugsgruppen vorherrschen, strukturiert. Dieses Zusammenspiel aus den vorhandenen strukturellen Bedingungen und der Auswahl durch den Menschen wird durch das Konzept des gesundheitsrelevanten Lebensstils verdeutlicht: Gesundheitsrelevante Lebensstile definieren wir als zeitlich relativ stabile typische Muster von gesundheitsrelevanten Verhaltensweisen, intrapersonellen und sozialen Ressourcen, welche von Individuen und Gruppen in Auseinandersetzung mit ihren sozialen, kulturellen und materiellen Lebensbedingungen entwickelt werden (Abb. 4.5).

Lebensstile unterstützen die Menschen bei verschiedenen Aufgaben im Rahmen ihrer Lebensgestaltung und Lebensbewältigung. Passend aufeinander abgestimmte Muster der Lebensführung können - oftmals unbewusst - die Identität des Einzelnen und das Gefühl der Zugehörigkeit zu bestimmten Gruppen stärken. Zudem können sie im Alltag hilfreiche Zeitstrukturen bilden sowie Entscheidungen des Alltags (z.B. Konsumentscheidungen) vorstrukturieren und erleichtern.

Für die Arbeit im Rahmen von Public Health ist das Konzept der Lebensstile besonders interessant, weil es hilft, gesundheitsrelevantes Verhalten in seiner sozialen Einbin- 


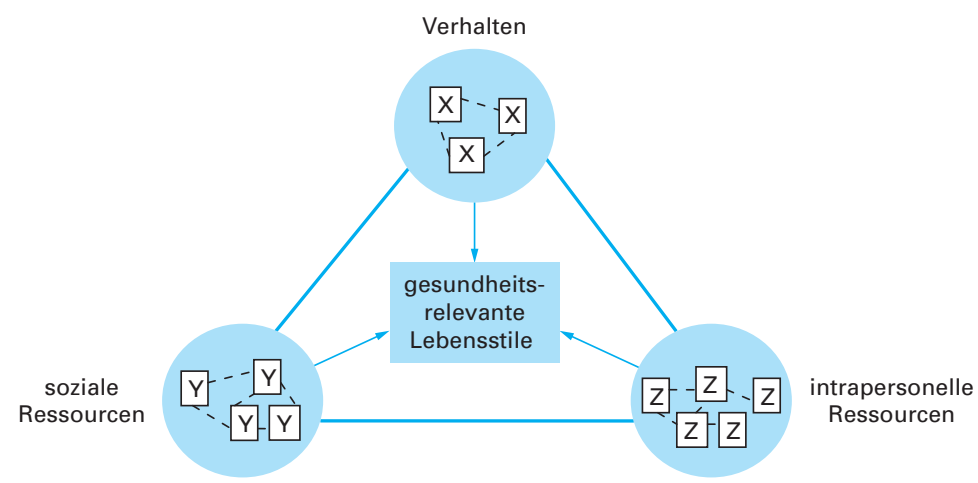

Abb. 4.5: Die drei Bereiche Verhalten, intrapersonelle und soziale Ressourcen sind konstituierende Dimension des Lebensstils. Sie stehen miteinander in Wechselwirkung und bilden sowohl in sich als auch untereinander ein aufeinander abgestimmtes, dynamisches Muster.

X: Verhaltensweisen wie Rauchen, körperliche Aktivität etc.

Y: Soziale Ressourcen wie gemeinschaftliche Unterstützung, Quartierangebote etc.

Z: Intrapersonelle Ressourcen wie Einstellungen, Gesundheitskompetenz etc.

dung zu verstehen. Dabei liefert es gleichzeitig wichtige Ansatzpunkte für Interventionen. Wenn risikoreiche Verhaltensweisen im Zusammenhang mit sozial geprägten Einstellungsmustern, den zur Verfügung stehenden Ressourcen sowie den Ansprüchen des Alltags verstanden werden, dann können sich Empfehlungen nicht einfach auf die Aufforderung zur Verhaltensumstellung beschränken. Zum Beispiel werden die Chancen für Interventionserfolge bei einer Gruppe von jungen Männern mit Risikoverhaltensweisen dann größer, wenn zusätzlich Verhältnisprävention betrieben wird, die es den Jugendlichen erleichtert, ihr Verhalten zu verändern. Folgendes Zitat des amerikanischen Public Health-Wissenschaftlers S. Leonard Syme verdeutlicht dies:

"We tend to study risk factors in individuals and we tend to focus interventions on individual behavior. The problem with this approach is that even if these interventions were completely successful, new people would continue to enter the at-risk population at an unaffected rate since we have done nothing to influence those forces in the community that caused the problem in the first place."

\subsubsection{Gesundheitskompetenz}

\section{Thomas Abel}

Zur Umsetzung von Lebensstiländerungen, d. h. Änderungen im Bereich des Verhaltens, der intrapersonellen und/oder sozialen Ressourcen, braucht es bestimmte Bedingungen im sozialen Kontext der Menschen. Zugleich bedarf es spezifischer individueller Kompetenzen der Menschen, damit diese ihre Verhaltensmuster und andere Aspekte ihres Lebensstils in einem gesundheitsförderlichen Sinne gestalten können. Hierzu gehören vor allem das Wissen und die Fähigkeiten im Umgang mit dem eigenen Körper, mit Gesundheit und Krankheit ebenso wie mit den gesundheitsprägenden sozialen Lebensbedingungen (s.a. soziale Determinanten, Kap. 4.1). Der hierfür heute zunehmend verwendete Begriff der Gesundheitskompetenz (Health Literacy) umfasst in einem wei- 
teren Sinn die individuellen Fähigkeiten, förderlich mit Gesundheit umzugehen. Dazu gehören ein Basisverständnis dessen, was die Gesundheit positiv oder negative beeinflusst sowie die Fähigkeit, Gesundheitsinformationen zu verstehen und sich im Versorgungswesen zurechtzufinden. Neben dem alltagspraktischen Wissen gehört zur Gesundheitskompetenz auch spezialisiertes Wissen, z. B. über individuelle und kollektive Gesundheitsrisiken, über den Umgang mit chronischen Einschränkungen oder zu spezifischen eigenen Erkrankungen bzw. Erkrankungen von Angehörigen.

In Hinblick auf ihre unterschiedlichen Anwendungen können drei Formen von Gesundheitskompetenz unterschieden werden:

- Funktionale Form: Hierzu gehören Grundfertigkeiten im Lesen und Schreiben, die im Umgang mit Gesundheit wichtig sind (z. B. das Verstehen von gesundheitsrelevanten Informationen).

- Interaktive Form: Dies sind zusätzliche kognitive und soziale Fertigkeiten, die zum Austausch von gesundheitsrelevanten Informationen und für praktische Hilfen nötig bzw. von Nutzen sind. Dazu gehören insbesondere die Beschaffung und der Austausch von Informationen sowie die Umsetzung der gewonnenen Informationen in den Lebensalltag. Beispiele: Das Sammeln von Informationen zu gesundheitsförderlichen Themen im sozialen Umfeld oder das Aneignen von spezifischerem Wissen zur eigenen Erkrankung im Gespräch mit der Ärztin.

- Kritische Form: Diese fortgeschrittenen kognitiven und sozialen Fertigkeiten ermöglichen es, gesundheitsrelevante Informationen kritisch zu analysieren, sodass sie im Sinne einer verbesserten Lebensbewältigung optimal genutzt werden können. Es beinhaltet auch die kritische Auseinandersetzung mit Empfehlungen für eine gesunde Lebensführung (z. B. die kritische Nachfrage beim Arzt oder die aufmerksame, differenzierte Betrachtung von Gesundheitsinformationen aus dem Internet).

Diese Kompetenzen werden primär über Kultur, Bildung und Erziehung erlernt und weitergegeben, aber auch über Austauschprozesse in spezifischen Situationen (z. B. im Arzt-Patient-Gespräch). Gesundheitskompetenz ist somit ein integrierter Bestandteil unserer kulturbasierten Ressourcen. Erwerb und Nutzung dieser Ressourcen werden stark durch den jeweiligen sozialen Hintergrund und die schichtenspezifische Lage eines Menschen geprägt. Eine gute Gesundheitskompetenz ermöglicht es einem Patienten, sich im oftmals komplexen Gesundheitssystem zurechtzufinden, die Angebote des Gesundheitsversorgungssystems zu nutzen sowie präventive und therapeutische Empfehlungen umzusetzen. Im Alltag der Menschen wirkt sich eine gute Gesundheitskompetenz primär als individuelle Ressource aus, die dazu beitragen kann, dass Menschen mehr Kontrolle über ihre Gesundheit und über gesundheitsbeeinflussende Faktoren (Gesundheitsdeterminanten, s. Kap. 4.1) erlangen. Im Idealfall führt eine verbesserte Gesundheitskompetenz sowohl zu einem individuellen Gesundheitsgewinn als auch zu besseren Rahmenbedingungen für die Gesundheit. Dabei ist es jedoch wichtig, Gesundheitskompetenz immer wieder zu fördern, da sich die Anforderungen an die Bevölkerung - insbesondere an PatientInnen - in einem sich schnell verändernden Gesundheitswesen laufend erhöhen. Beispiele für solche sich verändernden Anforderungen sind der Zugang zu Gesundheitsinformationen aus dem Internet oder die Unübersichtlichkeit im Bereich des Krankenversicherungsmarktes. 


\section{Internet-Ressourcen}

Auf unserer Lehrbuch-Homepage (www.public-health-kompakt.de) finden Sie Hinweise auf weiterführende Literatur sowie Links zu themenrelevanten Studien und Institutionen.

\subsection{Screening}

\section{Matthias Egger, Marcel Zwahlen, Angela Raffle}

Die Idee ist bestechend: Im Rahmen von Vorsorgeuntersuchungen wird bei Personen, die sich gesund fühlen festgestellt, ob ein frühes Stadium einer Erkrankung vorliegt. Der Krankheitsverlauf wird darauf durch die frühzeitig einsetzende Therapie günstig beeinflusst, sodass Komplikationen verhindert und die Sterblichkeit gesenkt werden. Ein solches Screening ist mehr als nur die Durchführung einer Vorsorgeuntersuchung. Es umfasst eine ganze Versorgungskette und sollte im Rahmen eines organisierten und evaluierten Screening-Programms stattfinden.

In diesem Abschnitt geben wir zuerst einem kurzen geschichtlichen Überblick und definieren dann den Begriff "Screening". Anschließend betrachten wir die Auswirkungen des Screenings und gehen auf mögliche Fallstricke bei der Evaluation von Screening-Programmen ein. Dabei zeigt sich, dass Screening nicht nur mit einem Nutzen, sondern immer auch mit unerwünschten Auswirkungen verbunden ist. Zum Schluss geben wir eine Übersicht über die in der Schweiz und in Deutschland durchgeführten Screening-Programme.

Schweizerische Lernziele: CPH 10-12

Die Idee, mit Hilfe von regelmäßigen Untersuchungen den Gesundheitszustand der Bevölkerung zu verbessern, entstand im 19. Jahrhundert. 1861 empfahl Dr. Horas Dobelle vom London Royal Hospital for Chest Diseases, dass Ärzte in bestimmten Abständen Untersuchungen bei ihren PatientInnen durchführen sollten, unabhängig davon, ob sie krank sind oder nicht. Diese Idee wurde in der Folgezeit in den USA von Lebensversicherungen und Arbeitgebern aus finanziellen Motiven aufgegriffen und von der Ärzteschaft gefördert. In Europa stand bei den Befürwortern solcher regelmäßigen Untersuchungen die Wehrdienst-Tauglichkeit von jungen Männern im Vordergrund. Die Untersuchungen waren anfangs unspezifisch. Zu Beginn führte man nur klinische Untersuchungen durch, später kamen Röntgenbilder der Lungen, Lungenfunktionstests, Elektrokarkardiogramme (EKG) sowie Bluttests und Tests auf okkultes Blut im Stuhl hinzu.

In den 1960er Jahren gerieten diese Untersuchungen dann zunehmend ins Kreuzfeuer der Kritik, da zwei große, randomisierte Studien (die Kaiser Permanente-Studie 1964 in den USA und die South East London-Studie 1967 in England) keinen Nutzen zeigen konnten. Die Gesamtsterblichkeit in der Gruppe der regelmäßig untersuchten Personen unterschied sich nicht signifikant von der Gesamtsterblichkeit in der Gruppe der nicht regelmäßig Untersuchten. Heute wird allgemein akzeptiert, dass Vorsorgeuntersuchungen und Screening-Programme umfassend evaluiert werden müssen, bevor sie eingeführt werden. Man hat inzwischen auch international akzeptierte Qualitätsstandards für 